\title{
Assessment of Newcastle disease vaccines from different veterinary outlets in
}

\section{Abeokuta}

\author{
${ }^{1}$ Oni, O. O., ${ }^{2}$ Bello, K. O. and ${ }^{1}$ Soyinka, O. F.
}

${ }^{I}$ Department of Veterinary Medicine and Surgery, ${ }^{2}$ Institute of Food security,

Environmental Resources and Agricultural Research,

Federal University of Agriculture Abeokuta, Nigeria

Abstract

Corresponding author: writewole@yahoo.com

Newcastle disease (ND) remains one of the major diseases ravaging the poultry industry in Nigeria. Vaccination of birds is carried out to protect birds against the disease. Despite vaccination against ND, birds still come down with the disease. This study was conducted to determine the potency of ND vaccines sold at different veterinary outlets in Abeokuta. Newcastle disease vaccines were purchased from three veterinary outlets (I, II and III) in Abeokuta over a period of 3 weeks and the haemagglutination (HA) titre determined. A total of 50 broiler chicks were also purchased and divided into 4 groups $A-D$. Groups $A-C$ had 12 birds each and vaccinated against ND while Group D (Control) had 14 birds and were not vaccinated against ND. Groups A-C were vaccinated with ND vaccines with different $H A$ titre and the antibody response determined using haemagglutination inhibition (HI) assay. Varying haemagglutination titre was recorded for all the vaccines purchased from the three outlets. The first batch of vaccines had haemagglutination titre of $0 \log _{2}, 6 \log _{2}$ and $0 \log _{2}$ for outlets $A, B$ and $C$ respectively. The second batch had $6 \log _{2}, 7 \log _{2}$ and $5 \log _{2}$ while the third batch had $4 \log _{2}, 3 \log _{2}$ and $3 \log _{2}$ for outlets $A, B$ and $C$ respectively. Antibody titre stimulated in vaccinated birds by the second batch of vaccines for groups $A, B$ and C birds were $1536 \log _{2}$, $1792 \log _{2}$ and $768 \log _{2}$ respectively, while the control birds had HI titre of $5 \log _{2}$. It is recommended that veterinary outlets improve the storage of vaccines, vaccine potency test be carried out on vaccines regularly and seromonitoring for humoral immune response in vaccinated chicken flocks be carried out for successful control of Newcastle disease.

Keywords: Vaccination, seromonitoring, potency, haemagglutination

\section{Introduction}

Newcastle disease (ND) is an acute infectious and highly contagious disease (Ohore et al., 2002) with the potential of causing $100 \%$ morbidity and mortality in unvaccinated poultry (Chakrabarti et al., 2006). The disease has both epizootic and enzootic patterns in different flocks or population but it is an epizootic in intensive poultry and is responsible for most economic losses associated with poultry production (Diel et al., 2012). The occurrence of highly virulent ND infections are recognized as notifiable disease reportable to the Office of International Epizooties (Miller et al., 2015) and is one of the main sanitary barriers for the free trade of poultry and poultry products (Chitate and Gutal, 2011). Newcastle Disease in Nigeria has been a limiting factor to poultry meat and egg production (Saidu et al., 1994). The severity of the disease is dependent on the host age, immune status of the birds, environmental stress and on the virulence of the strain of the Newcastle disease virus (McFerran et al., 1988). In the control of this disease bio-security and hygiene measures are very essential in the prevention of the introduction or the spread of the disease.

In addition to bio-security measures, the 


\section{Assessment of Newcastle disease vaccines}

disease can be controlled by vaccination.

Vaccines are biological substances given to animals through specified routes and in correct dosage to protect against disease via stimulation of active immune response (Merchant et al., 2005). Vaccination is an important effort towards the control of ND (Alexander, 2001). The use of viable nonpathogenic isolates of Newcastle disease virus to immunize poultry against pathogenic strains of the virus has been a common practice since the B1 strain was first described in 1948. Numerous ND viruses with different levels of pathogenicity have been used to achieve desired immunologic response, using different vaccination regimes (Emikpe et al., 2007). However, despite vaccination, the disease continues to ravage the Nigerian poultry industry and thus suggests vaccination failure. Vaccine failure could be as a result of so many factors including; improper vaccine storage and transportation, improper administration, insufficient vaccine antigen, handling of the vaccines due to transport difficulties, high ambient temperatures, lack of refrigeration, non-relatedness to the field strain and poor management of flocks or the presence of inter-current disease (Dortmans et al., 2012, Miller et al., 2013). In addition to these, the epileptic power supply in Nigeria could also be a contributing factor to breakage in cold chain of vaccine during storage especially in veterinary outlets that are wholly dependent on power supply from the national grid. In Nigeria, where there is little information as to the efficacy of most commonly employed ND vaccination regimes, farmers tend to repeat vaccination at relatively short intervals due to uncertainty of protective immune profile. The need to evaluate the field situation as regards the effectiveness of some available ND vaccines in the market is thus expedient. This study was aimed at determining the titre of Newcastle Disease vaccines from different veterinary outlets pre-vaccination and antibody titre stimulated by such vaccines in broiler birds post vaccination.

\section{Materials and methods Vaccines}

Newcastle disease vaccines (lasota, komarov) were purchased from 3 randomly selected veterinary outlets in Abeokuta. Vaccines were purchased on a weekly basis from the three outlets. After each purchase, they were transported to the laboratory in ice packs to maintain their cold chain and analysed on the same day of purchase. The date of purchase, manufacturer, manufacturing date, expiry date and batch numbers were noted. In all, 9 vials of Newcastle disease vaccines were purchased over a period of 6 weeks for which broiler birds were raised.

\section{Experimental birds}

All protocols for this research were approved by the Animal Care and Use Committee of the Federal University of Agriculture, Abeokuta, Nigeria. Broiler chicks were vaccinated with ND vaccine using the schedule in table 1 . The broiler birds were maintained at the poultry Unit of College of Veterinary Medicine, Federal University of Agriculture, Abeokuta. The birds were divided into four groups A, B, C and D. Groups A, B and C were vaccinated with Newcastle Disease vaccines from the 3 Veterinary outlets while group D birds were not vaccinated against ND and served as the control group. Birds were raised in a controlled environment with feed and water supplied ad-libitum.

\section{Collection of sera for haemagglutination inhibition test}

A total of 5 chicks were randomly selected from the birds before vaccination to 


\section{Oni, Bello and Soyinka}

determine maternal antibody level in the chicks. After vaccination blood samples were collected using $2 \mathrm{ml}$ needle and syringe on a weekly basis before a booster dose of ND vaccine was administered when necessary. The blood samples were allowed to clot in bijou bottles and sera were separated and collected into $1.5 \mathrm{ml}$ microcentrifuge tubes, then labeled accordingly. Sera samples were frozen until analyzed using the Haemagglutination and haemagglutination inhibition.

\section{Haemagglutination (HA) Test Washing} and standardization of $R B C$ suspension

The packed cell volume of the blood sample collected from healthy chicken was determined using heamatocrit reader before washing. The value obtained was used in preparing $0.5 \%$ RBC suspensions to determine the HA.

\section{Haemagglutination inhibition (HI) Test}

The HI test was performed using the procedure (constant virus and varying serum) against 4HA units of the ND LaSota virus as described by Anosa and Adene (2007).

\section{Results}

Table 1 shows the result of HA test used to determine the potency of Newcastle disease vaccine from different veterinary outlets. The antibody titre stimulated in broiler birds by the vaccines is as shown table 2 and graphically presented in figure 1 .

Table 1: HA titre of Newcastle Disease vaccines from different veterinary outlets

\begin{tabular}{clll}
\hline Week & Outlet A & Outlet B & Outlet C \\
\hline 1 & Lasota $\left(0 \log _{2}\right)$ & Lasota $\left(6 \log _{2}\right)$ & Lasota $\left(0 \log _{2}\right)$ \\
2 & Lasota $\left(6 \log _{2}\right)$ & Lasota $\left(7 \log _{2}\right)$ & Lasota $\left(5 \log _{2}\right)$ \\
3 & R2B $\left(4 \log _{2}\right)$ & R2B $\left(4 \log _{2}\right)$ & R2B $\left(4 \log _{2}\right)$ \\
\hline
\end{tabular}

Table 2: HI result of birds vaccinated with Newcastle disease vaccine from different veterinary outlets.

\begin{tabular}{ccccc}
\hline Age (days) & Group A & Group B & Group C & Group D \\
\hline 4 & 72 & 72 & 72 & 72 \\
13 & 64 & 72 & 64 & 64 \\
19 & 51.2 & 51.2 & 51.2 & 51.2 \\
27 & 32 & 28.8 & 57.6 & 19.2 \\
31 & 288 & 704 & 288 & 12 \\
38 & 384 & 640 & 384 & 6 \\
53 & 1536 & 1792 & 768 & 5 \\
\hline
\end{tabular}

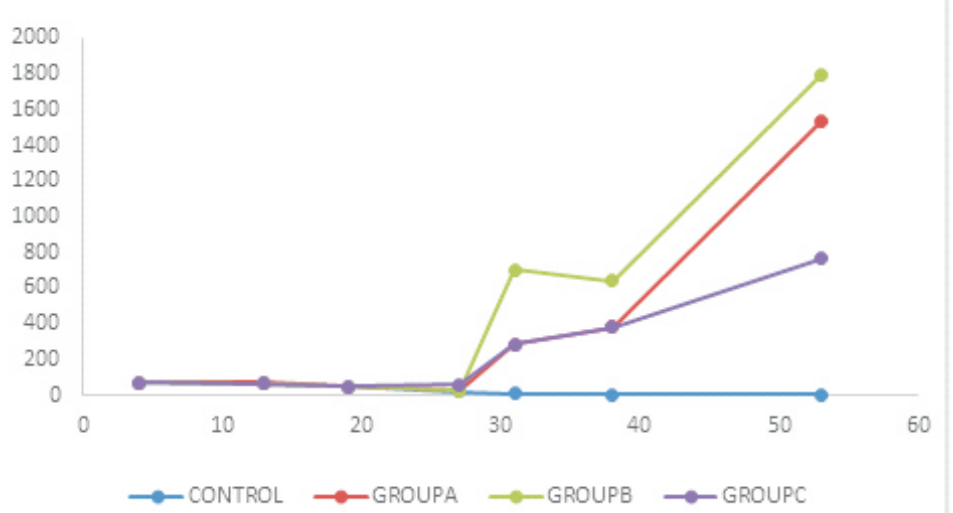

Figure 1: Line graph representation of antibody titre in Newcastle vaccination. 


\section{Assessment of Newcastle disease vaccines}

\section{Discussion}

Among the infectious diseases, Newcastle disease remains a deadly viral disease of poultry with high contagiousness and rapid spreading among chicken, domestic and semidomestic species of birds (Rahman et al., 2002). It is thus important that vaccines stimulate adequate antibody titre to protect them from the disease.

In this study, result of ND vaccine purchased from the three outlets revealed varying vaccine titre for different days. Although, some vaccines were from the same manufacturer the titre were different in the same veterinary outlet. The first batch of vaccines purchased from outlets $\mathrm{A}, \mathrm{B}$ and $\mathrm{C}$ had haemagglutination titre of $0 \log _{2}$, $6 \log _{2}$ and $0 \log _{2}$ respectively as shown in table 1 . The implication of these results is that vaccines from outlets $\mathrm{A}$ and $\mathrm{C}$ would not stimulate any immune response in birds because the titre had waned. Vaccine from outlet $\mathrm{B}$ however had a higher titre for better stimulation of immune response. There are various reasons why the titre of these vaccines would be very low. These includes: poor storage, break in cold chain (Abbas et al., 2006), freezing and thawing. The continuous maintenance of a cold chain is difficult for most of the veterinary outlets in Abeokuta due to the epileptic power supply. So, these outlets result to storing vaccines in coolers using ice blocks or in freezers without regular power supply. This is not ideal, as the refrigerator temperature of $+2^{\circ} \mathrm{C}$ to $+4^{\circ} \mathrm{C}$ which vaccines require would not be maintained continuously. For live vaccines to work as they were designed, they must be stored, mixed, dosed and applied appropriately (Bermudez et al., 2003). They also reported that vaccines generally are licensed based on protection studies performed in specific pathogen free type leghorn birds, absent of any circulating antibody to that particular agent, at the youngest age on the label, and at the minimum titre expected at the end of the dating period allowed for each given vaccine. The second batch of vaccines had a higher HA titre than the first batch with outlet B having the highest HA titre of $7 \log _{2}$ as shown in Table 1. Low vaccine titre was also seen in the third batch of vaccines from the three outlets. The third batch of vaccines from the three outlets was all from the same manufacturer. The HA titre of the vaccines were however different for outlet A, B and C having $4 \log _{2}, 3 \log _{2}$ and $3 \log _{2}$ respectively. It is expected that if properly stored, these vaccines should have the same HA titre. Although, vaccines from outlet $B$ and $C$ had the same HA titre, it was lower than outlet A. Veterinarians and in some cases farmers who purchased these vaccines, are not aware if a vaccine is potent or not. They innocently vaccinate birds with vaccines with little or no titre as the case may be. However, birds still come down with the disease because such vaccines do not stimulate enough immune response in the birds. It is thus important that vaccine potency be determined before it is administered and storage should also be properly carried out.

The result from the HI test indicate that the birds responded to the vaccines according to the titre of the vaccine administered (Table 2 ). The antibody level in all the groups continued to wane up to the fourth week of life. From this point onward, a rise in the antibody titre was seen in birds of groups A$\mathrm{C}$ after vaccination. The control group D however, remained low because they were not vaccinated. After 7 weeks of vaccination, groups $\mathrm{A}, \mathrm{B}, \mathrm{C}$ and $\mathrm{D}$ had $\mathrm{HI}$ titre of $1536 \log _{2}, \quad 1792 \log _{2}, 768 \log _{2}$ and $5 \log _{2}$ respectively. Group B birds vaccinated with $7 \log _{2}$ titre vaccine had the highest antibody titre followed by group A 


\section{Oni, Bello and Soyinka}

and then $\mathrm{C}$ as shown in figure I. This further confirms that the lower the vaccine titre the lower the immune response stimulated in the birds, although all the vaccinated birds were adequately protected.

The most reliable control method for Newcastle disease is by vaccination. It is thus recommended that potency of ND vaccine be determined before vaccination of birds especially in locations with epileptic power supply. The potency of a vaccine determines the amount of antibody that will be stimulated by the birds in the absence of maternally derived antibody. In addition, protection for most of live vaccines does not last for a full breeder cycle and needs regular revaccination (Roy et al., 2003) especially when birds are not adequately stimulated with a potent vaccine. Serologic test and seromonitoring for humoral immune response in vaccinated chicken flocks is necessary for controlling Newcastle disease. It is advised that farmers and marketers perform these tests in order to know the status of flocks and vaccines for sale respectively. Also, farmers should consult veterinarians if any vaccine is to be administered on a farm. This is not only to aid proper administration but also to determine vaccine titre where possible.

\section{References}

Abbas, A., Muneer, M. A., Ahmed, M. D., Khan, M. A., Youns, M. and Khan, I. 2006. C o $\mathrm{m}$ p a r a t i v e efficacy of five different brands of commercial Newcastle disease Lasota virus vaccines in broilers. Pakistan Vet. J. 26(2):5558.

Alexander, D. J. 2001. Newcastle disease. The Gordon Memorial Lecture. British. Poult. Sci. 42:5-22.

Anosa, G. N., Adene, D. F. 2007. The comparative immunogenicity of three lentogenic brands of Newcastle disease vaccines in Nigeria. Nigerian Vet. J. 28(2):1-5.

Awang, I. P. R., Wan, W. S. and Abdurazak, J. 1992. Detection of maternal antibodies against Newcastle disease virus in chicks $\mathrm{u} \mathrm{s}$ i n $\mathrm{g}$ a $\mathrm{n}$ i $\mathrm{nd}$ i r e c t immunoperoxidate test. J. Vet. Med. 4: 19-23.

Bermudez, A. J. and Stewart-Brown, B. 2003. Disease prevention and diagnosis. In Diseases of poultry (Y.M. Saif, ed.), 11th Ed. Iowa State University Press, Ames, 1754.

Chakrabarti, S., King, D. J., Cardona, C., Afonso, C. L., Swayne, D. E. and Gerry, A. C. 2006. Potential role of flies in the persistence and dispersal of exotic Newcastle disease virus in Southern California. Livestock Insect Worker's Conference Annual Meeting. p. 34.

Chitate, F. and Gutal, M. 2011. Country Report: Zimbabwe. Retrieved from http://aciar.gov.au/files /node/2131/pr103chapter12zimba bwe.pdf

Diel, D. J., Susta L., Garcia, S. C., Killian, M. L., Brown, C. C., Miller, P. J. and Afonso, C. L. 2012. Complete genome and clinico-pathological characterization of a virulent newcastle disease virus isolate from South America. J. Clin. Microbiol. 50(2):378-387.

Dortmans, J. C., Peeters, B. P., Koch, G. 2012. Newcastle disease virus outbreaks: vaccine mismatch or inadequate application? Vet. Microbiol. 160: 17-22.

Emikpe, B. O., Oladele, O. A., Ikubor, A. 


\section{Assessment of Newcastle disease vaccines}

O. and Ockiya, M. A. 2007. The laboratory assessment of common Newcastle vaccination regimes in use in Nigerian Poultry. $J$. of Ani.and Vet. Adv. 6: 971-974.

Mcferran and McCracken 1988. Newcastle disease. D.J (ed.). Kluwer academics Publishers, Boston. Massachusetts. pp 161-183.

Merchant, I. A., Packer, R. A. 2005. Newcastle disease virus lentogenic vaccines against virulent Asiatic type Newcastle disease viruses. Veterinary bacteriology and virology $7^{\text {th }}$ edition pp. 670-674. Poultry Science Association, Inc. 12:169-173.

Miller, P. J., Haddas, R., Simanov, L., Lublin, A., Rehmani, S. F., Wajid, A., Bibi, T., Khan, T. A., Yaqub, T., Setiyaningsih, S. and Afonso, C. L. 2015. Identification of new sub-genotypes of virulent Newcastle disease virus with potential panzootic features. Infect Genet. Evol. 29: 216-29. http://dx.doi.org/10.1016/j.meegid .2014 .10 .032 . d o i : 10.1016/j.meegid.2014.10.032PM ID: 25445644

Miller, P. J., Afonso, C. L., Attrache, J. E., Dorsey, K. M., Courtney, S. C., Guo, Z. and Kapezynski, D. R. 2013. Effects of Newcastle disease virus vaccine antibodies on the shedding and transmission of challenge viruses. Dev. and Comp. Immunol. 41:505-513
Ohore, O. G., Ozegbe, P. C., Emikpe, B. O., Okojie, V. E. 2002. Survey of antibodies to Newcastle disease virus in apparently healthy adult Nigerian indigenous chickens (Gallus domesticus) in Ibadan using ELISA. African Journal of Clinical and Experimental Microbiology 3(1): 38-40.

Rahman, M. M., Bar, A. S. M., Giasuddin, M., Islam, M. R., Alam, J., Sil, G. C. and Rahman, M. M. 2002. Evaluation of maternal and humoral immunity against Newcastle disease virus in chicken. Interna. J. Poul. Sci. 2002;1(5):161-163.

Roy, P., Venugopalan, A. T. and Dhillon, A. S. 2003. Efficacy of two commercial Newcastle disease virus lentogenic vaccines against virulent Asiatic-Type Newcastle disease viruses. J. Appl. Poult. Res. 12: $169-173$

Saidu, L., Nwangu, B. I. and Otchore, E. O. 1994. Disease of Nigerian indigenous chicken. Bulletin of Animal Health and Production in Africa, 42, 17-28.

Received: $11^{\text {th }}$ March, 2017 Accepted: 21 ${ }^{\text {st }}$ June, 2017 